\title{
New Manifolds for Superstring Compactification
}

\author{
Andrew Strominger ${ }^{1}$ and Edward Witten ${ }^{2}$ \\ 1 The Institute for Advanced Study, Princeton, NJ 08540, USA \\ 2 Princeton University, Princeton, NJ 08544, USA
}

\begin{abstract}
We construct new manifolds with SU(3) holonomy that are candidate vacua for superstring theory and give a detailed explanation of the techniques involved. Some of these manifolds have a non-abelian fundamental group and thus can lead to a rank five low energy gauge group. Models with an odd number of generations are obtained. $\mathscr{C} \mathscr{P}$ violation is discussed in this context and shown to occur generically. Topological features of the Yukawa couplings are also discussed.
\end{abstract}

\section{Introduction}

The discovery of a generalized mechanism of anomaly cancellation [1] has made superstring theory [2] not just a logically viable approach to quantum gravity but also a practical approach to phenomenology. Especially promising is the new superstring theory with $E_{8} \times E_{8}$ gauge group [3]. One approach to phenomenology [4] is to consider compactification from ten dimensions to $M^{4} \times K, M^{4}$ being four dimensional Minkowski space and $K$ being a six dimensional CalabiYau manifold, i.e. a manifold of SU(3) holonomy. These manifolds are (at least in tree approximation) vacuum solutions of the string theory. Supersymmetry is preserved at the Planck scale, which may be useful for solving the hierarchy problem. The gauge field acquires an expectation value on $K$, and the gauge group is broken down to $E_{6} \times E_{8}$. Ordinary fermions appear in the 27 of $E_{6}$. The number of generations is $\frac{1}{2}|\chi|, \chi$ being the Euler characteristic of $K$. Properties of these models have been further investigated in several recent papers [5-11].

It is beyond present technology to determine from the string theory which of the many Calabi-Yau manifolds, if any, indeed represents the true ground state of the theory. However, phenomonological considerations severely limit the possibilities. For example [4], in order to get a reasonably small number of generations, one must find a Calabi-Yau manifold with small $\chi$.

Most straightforward constructions of Calabi-Yau manifolds seem to give simply connected manifolds with rather large $|\chi|$. However, $\chi$ can be reduced by passing from $K$ to $K / G$, where $G$ is a discrete symmetry group of $K$ that acts freely. 
In [4], a model with $\chi=-8$, and therefore with four generations, was constructed in this way. Recently, more detailed investigations of phenomenological implications have produced further constraints on the internal manifold, probably ruling out the four generation model of ref. [4]. One constraint arises from GeorgiQuinn-Weinberg type renormalization group calculations. Since $E_{6}$ unification necessarily occurs at the compactification scale, the requirement that the strong and electroweak couplings have their observed values at low energies is quite stringent (and appears to favor a three generation model) [6]. A second constraint comes from proton decay. In general, supersymmetric $E_{6}$ models have rapid proton decay mediated by scalar quarks and leptons. These can be eliminated by a discrete symmetry that forbids the troublesome Yukawa couplings. Although discrete symmetries abound in Calabi-Yau compactifications, one with exactly the required properties has not been found. It is thus imperative to find more examples of Calabi-Yau manifolds and to investigate their properties.

The main purpose of the present paper is to construct other examples of Calabi-Yau manifolds with a reasonably low number of generations. In fact, we will construct examples with one, two, and four generations. The one generation case (described in Sect. III) could not be the real world, but it is an interesting theoretical laboratory, especially since it shows that the number of generations is not always even. Two generation $E_{6}$ models are nearly capable of describing known phenomena [12], but data on b quark decay probably rules out this approach [13]. Four generation models are compatible with most observations (the fourth generation could well have a mass of order $M_{W}$ ), but may be disfavored by renormalization group calculations. We have not managed to find a three generation model, although the fact that models exist with an odd number of generations suggests that three generations is possible.

The organization of this paper is as follows. In Sect. II, we construct new examples with two or four generations by the method used in [4] - dividing a simply connected manifold by the free action of a discrete group. In Sect. IV, we use a more general technique (dividing by discrete symmetries that have certain types of fixed points) to construct examples with one, two, or four generations. In Sect. IV we discuss the equivalence of the existence of a holomorphic three form to the vanishing of the first Chern class $\left(c_{1}\right)$ and show how this fact can be used to determine when $c_{1}=0$. In Sect. $\mathrm{V}$, we describe how manifolds of SU(3) holonomy can be constructed by taking suitable branched covers of manifolds of U(3) holonomy. In Sect. VI we turn to the question of $\mathscr{C} \mathscr{P}$ violation in Calabi-Yau manifolds. We will see that $\mathscr{C} \mathscr{P}$ violation is possible in nearly all Calabi-Yau manifolds. In Sect. VII, we discuss general formulas for Yukawa couplings and their topological properties.

A noteworthy feature of the manifolds constructed here is that several of the two and four generation models have non-abelian fundamental group. This makes it possible [5] for the low energy gauge group in four dimensions to be a rank five group (such as $\mathrm{SU}(3) \times \mathrm{SU}(2) \times \mathrm{U}(1) \times \mathrm{U}(1)$ ) rather than a rank six group.

\section{Free Actions of Discrete Groups}

One of the models considered in $[4]$ was $Y(7 ; 2,2,2,2)$, the intersection of four quadrics in $C P^{7}$. Thus, let $z_{i}, i=1, \ldots, 8$, be eight complex variables, not all zero, 
with $\left\{z_{i}\right\}$ and $\left\{\lambda z_{i}\right\}$ considered equivalent for any non-zero $\lambda$. Let $I_{k}, k=1, \ldots, 4$, be four homogeneous quadratic polynomials in the $z_{i}$, which obey a transverse intersection condition described below. Then the equation $I_{1}=I_{2}=I_{3}=I_{4}=0$ defines a manifold $K$ of complex dimension three and vanishing first Chern class. It has Euler characteristic $\chi=-128$, corresponding to 64 generations. In [4], it was mentioned that $K$ (for suitable $I_{k}$ ), can admit the free action of $Z_{8}$ or $Z_{2} \times Z_{2} \times Z_{2}$. The model obtained in this way is an eight generation model. In this section we will describe several examples of groups with 16 or 32 elements that can act freely on the intersection of four quadrics, giving models with two or four generations.

It is not enough to find an intersection of quadrics $I_{k}=0, k=1, \ldots, 4$, on which a group $G$ acts freely. It is also necessary to verify that, in the language of algebraic geometry, the intersection of quadrics is "transverse," i.e. that the equation $I_{k}=0$ defines a smooth, non-singular manifold. The criterion for a transverse intersection is that the four form $\omega=d I_{1} \wedge d I_{2} \wedge d I_{3} \wedge d I_{4}$ does not vanish at any point where all $I_{k}=0 .{ }^{1}$ At any point where $\omega \neq 0$, there is a non-singular local holomorphic change of coordinates from the independent variables $z_{1}, \ldots, z_{7}\left(z_{8}\right.$ can be set to 1 by scaling) to new variables consisting of the $I_{k}$ and three independent variables $y_{1}, y_{2}, y_{3}$. In these coordinates, the locus of $I_{k}=0$ is a nonsingular space spanned locally by the $y_{i}$. If the equation $0=\omega=I_{k}$ has a simultaneous solution, the manifold defined by $I_{k}=0$ is singular at that point. The equations $0=\omega=I_{k}$ are overdetermined (more than seven equations for the seven unknowns), so generically they have no solution. However, when we start to restrict the $I_{k}$ by global symmetries, we are not dealing with a generic set of quadrics, and it is necessary to check the transverse intersection condition carefully.

To try to find models with large freely acting symmetries, we first imitate the model in [4] and try to find a model with a $Z_{8} \times Z_{8}$ symmetry. Let $\mathrm{S}$ and $\mathrm{T}$ be defined by $S\left(z_{i}\right)=z_{i+1}, T\left(z_{i}\right)=\beta^{i} z_{i}$, where $\beta=\exp (2 \pi i / 8)$, and we identify $z_{i+8}$ with $z_{i}$. Then $\mathrm{S}$ and $\mathrm{T}$ generate a group $G$ isomorphic to $Z_{8} \times Z_{8}{ }^{2}$ The following set of four quadrics is invariant under $G$.

$$
I_{v}=\frac{1}{2} \sum_{k=1}^{8} z_{k}^{2} \beta^{2 k v}+\varepsilon \sum_{k=1}^{8} z_{k-1} z_{k+1} \beta^{2 k v}+\frac{\delta}{2} \sum_{k=1}^{8} z_{k-2} z_{k+2} \beta^{2 k v}, \quad v=0,1,2,3 .
$$

Here $\varepsilon$ and $\delta$ are arbitrary complex parameters. The individual $I_{v}$ are not invariant under $Z_{8} \times Z_{8}$. T permutes the $I_{v}$, and $\mathrm{S}$ transforms $I_{v} \rightarrow \beta^{-2 v} I_{v}$. But the locus $K$ of $I_{v}=0, v=0, \ldots, 3$ is invariant under $Z_{8} \times Z_{8}$.

It is not difficult to check that $Z_{8} \times Z_{8}$ acts on $K$ without fixed points. It must be checked that $S^{k} T^{l}$ has no fixed points on $K$ unless $k$ and $l$ are multiples of 8 . For any element $x$ of a finite group, there is an integer $n$ called the order of $x$ defined as the lowest positive integer such that $x^{n}=1$. In $Z_{8} \times Z_{8}$ any element has order $2^{r}$ for

1 To get a heuristic feeling for this criterion, consider a polynomial $I(x, y)$ in two real variables $x$ and $y$. The equation $I(x, y)=0$ describes a curve in the $x-y$ plane which is non-singular if $I=d I=0$ has no solution. For instance, $I(x, y)=x-y$ corresponds to a line. If $I(x, y)=x^{2}-y^{2}$, then $I=d I=0$ at $x=y=0$, and the curve defined by $I=0$ (which consists of two lines) is singular at $x=y=0$ (where the two lines intersect)

2 Actually $S T=T S \beta^{-1}$. In $C P^{7}$, the phase $\beta^{-1}$ is irrelevant, so $S T=T S$, and the group is $Z_{8} \times Z_{8}$. Similar remarks hold for many examples considered later 
some $r$. If $S^{k} T^{l}$ has order $2^{r}$, then $\theta=\left(S^{k} T^{l}\right)^{2^{r-1}}$ has order 2 . If $\theta$ has no fixed points $S^{k} T^{l}$ has none (since a fixed point of $S^{k} T^{l}$ is certainly a fixed point of $\theta$ ). Hence to show that $Z_{8} \times Z_{8}$ acts freely, it is enough to show that the elements of order two act freely. The elements of $Z_{8} \times Z_{8}$ of order 2 are $S^{4}, T^{4}$ and $S^{4} T^{4}$. It is easy to check that for generic $\varepsilon, \delta$ they have no fixed points. The "reason" for this is that the $Z_{8} \times Z_{8}$ action has been chosen so that each element $x$ of order 2 has four eigenvalues +1 and four -1 when acting on the $z_{i}$. The fixed point set of $x$ in $C P^{7}$ consists then of two copies of $C P^{3}$, each of dimension three. Also, the quadratic equations have been chosen to be invariant under the elements of order two. Under these conditions the fixed point set of the $x$ 's can be expected not to intersect the four quadrics, since four equations for three unknowns generically have no solution.

We now must check whether the quadrics defined by $I_{v}=0$ intersect transversely. If so, the space $K /\left(Z_{8} \times Z_{8}\right)$ would have Euler characteristic - 2 , and hence one generation. However, it can be shown that the intersection is not transverse; the equations $I_{0}=I_{1}=I_{2}=I_{3}=d I_{0} \wedge d I_{1} \wedge d I_{2} \wedge d I_{3}=0$ have a solution at $z_{0}=z_{4}=0, z_{2}=z_{6}$, for certain values of the other variables.

If we consider instead the group $Z_{8} \times Z_{4}$ generated by $S$ and $T^{2}$, the conclusion is different. We can now consider the following set of four quadrics:

$$
I_{v}=\frac{1}{2} \sum_{k=1}^{8} z_{k}^{2} \beta^{2 k v}+\varepsilon \sum_{k=1}^{8} z_{k-1} z_{k+1} \beta^{2 k v}\left(1+\lambda(-1)^{v}\right)+\frac{\delta}{2} \sum_{k=1}^{8} z_{k-2} z_{k+2} \beta^{2 k v}
$$

Here $\varepsilon, \lambda$, and $\delta$ are complex parameters. The group action on the intersection $K$ of the quadrics is free, as it was previously. However, now the intersection of the quadrics $I_{v}=0$ can be shown to be transverse, for generic $\varepsilon, \lambda$, and $\delta$. Hence, $K /\left(Z_{8}\right.$ $\times Z_{4}$ ) is a model with $\chi=-4$ and two generations. (Note $[4,5]$ that three complex parameters $\varepsilon, \lambda$, and $\delta$ is the proper number for a Calabi-Yau manifold with $b^{1,1}=1$ and $\chi=-4$.) A slight variant of this gives some new four generation models. Let $H$ be the $Z_{4} \times Z_{4}$ group generated by $S^{2}$ and $T^{2}$, the $Z_{8} \times Z_{2}$ group generated by $S$ and $T^{4}$, or the $Z_{8} \times Z_{2}$ group generated by $S T^{2}$ and $T^{4}$. Then $K / H$ has $\chi=-8$ and four generations. Of course, in any of these cases two more complex parameters can be included in (2.2).

We will not here carry out the detals of proving that the quadrics $I_{v}=0$ intersect transversely for generical $\varepsilon, \lambda$, and $\delta$; the analogous calculation in a similar (but somewhat simpler) case is described below. However, a few general comments may be useful. To prove that the intersection is transverse generically for almost all $\varepsilon, \lambda$, and $\delta$, it is enough to show the intersection is transverse for some $\varepsilon, \lambda$, and $\delta$. For, as $C P^{7}$ is compact, if the equations $I_{0}=I_{1}=I_{2}=I_{3}$ $=d I_{0} \wedge d I_{1} \wedge d I_{2} \wedge d I_{3}=0$ have no solution on $C P^{7}$, they fail to have a solution by some minimum amount $\Delta$. Hence, if $\varepsilon, \lambda$, and $\delta$ are perturbed slightly, these equations still have no solution. Therefore, if the set of $(\varepsilon, \lambda, \delta)$ for which the intersection is transverse is not empty, it contains an open set. By analytic continuation, it follows then that the intersection is transverse for almost all $(\varepsilon, \lambda, \delta)$. Hence, it is natural to try to find some special values of $\varepsilon, \lambda, \delta$ for which the intersection is transverse. One might try to set $\varepsilon$ or $\lambda$ or $\delta$ to zero, but all of these attempts are easily seen to fail. An alternative which does work is to regard $\varepsilon$ as infinitesimal and systematically drop from the equations all terms of order $\varepsilon^{2}$. It 
can be shown that in this approximation the equations $0=I_{v}$ $=d I_{0} \wedge d I_{1} \wedge d I_{2} \wedge d I_{3}$ have no solution (for generic $\lambda$ and $\delta$ ). Hence, $K /\left(Z_{8} \times Z_{4}\right)$ is non-singular for almost all $(\varepsilon, \lambda, \delta)$. The same is certainly also true for $K / H$, which is a double cover of $K /\left(Z_{8} \times Z_{4}\right)$.

Now we will construct a two generation model whose fundamental group is not $G=Z_{8} \times Z_{4}$ but a non-abelian group $G^{\prime}$ with 32 elements. Let $W$ be defined by $W: z_{i} \rightarrow z_{3 i+1}$. $W$ acts freely on $K$ since $W^{2}=S^{4}$, and we already know that $S^{4}$ acts freely. $W$ and $S^{2}$ generate a nonabelian group $Q$ with eight elements which can be identified as the group of "unit quaternions" whose elements are $\pm 1, \pm i \sigma_{1}, \pm i \sigma_{2}$, and $\pm i \sigma_{3}$. This may be readily seen if the eight $z_{i}$ are identified with the unit quaternions in the following way,

$$
\begin{array}{cccccccc}
z_{1} & z_{2} & z_{3} & z_{4} & z_{5} & z_{6} & z_{7} & z_{8} \\
1 & i \sigma_{1} & i \sigma_{2} & i \sigma_{3} & -1 & -i \sigma_{1} & -i \sigma_{2} & -i \sigma_{3}
\end{array},
$$

then the action of $S^{2}$ and $W$ correspond to left multiplication of the unit quaternions by $i \sigma_{2}$ and $i \sigma_{3}$, respectively. Since $Q$ acts freel,y $K / Q$ is an eight generation model.

Although $S$ and $W$ generate a group with sixteen elements, this group does not act freely (it contains the element of order two $S^{-1} W$, which has fixed points). However, $S^{2}, W$, and $T^{2}$ generate a 32 element symmetry group $G^{\prime}$, since $W T^{2} W^{-1} T^{-1}=i T^{4}$ and $S^{2} T^{2} S^{-2} T^{-2}=-1 . G^{\prime}$ acts freely (the elements of order two are $S^{4}, T^{4}$, and $S^{4} T^{4}$ ), so $K / G^{\prime}$ is another two generation model. Again, various double coverings of $K / G^{\prime}$ give models with four generations.

The group $G^{\prime}$ has irreducible two dimensional unitary representations. (For instance, one can represent $T^{2}$ by 1 and $\sigma_{i}$ by the usual Pauli spin matrices.) Hence [5], ten dimensional supergravity compactified on $M^{4} \times K / G^{\prime}$ can give a rank five group at low energies.

At first sight, it is rather surprising to find inequivalent models $K / G, K / G^{\prime}$, with the same universal covering space $K$. Their physical properties are quite different; for instance, $K / G^{\prime}$ leads to low energy gauge groups of rank five or rank six, but $K / G$ leads only to rank six groups. Elements of $G^{\prime}$ that are not in $G$ are global symmetries of $K / G$, and vice-versa. (Thus, $W$ is a global symmetry of $K / G$, and $S$ is a global symmetry of $K / G^{\prime}$.) Since these symmetries do not act freely, one cannot divide by them to get a one generation model, but they still restrict Yukawa couplings. (Thus, $W$ invariance restricts Yukawa couplings on $K / G$, and $S$ invariance restricts Yukawa couplings on $K / G^{\prime}$ ). More generally, Yukawa couplings on, say, $K / G$ can be limited by "pseudosymmetries" that are symmetries only of the covering space $K$ and not of $K / G$.

Before leaving this example, let us pause to rewrite Eq. (2.2) in another form that is in some respects simpler. Let $J_{l}=\frac{1}{4} \sum_{v} I_{v} \beta^{-2 l v}, l=0, \ldots, 3$. Explicitly,

$$
\begin{aligned}
& J_{0}=\frac{1}{2}\left(z_{0}^{2}+z_{4}^{2}\right)+\varepsilon\left(z_{1} z_{7}+z_{3} z_{5}\right)+\varepsilon \lambda\left(z_{1} z_{3}+z_{5} z_{7}\right)+\delta z_{2} z_{6}, \\
& J_{1}=\frac{1}{2}\left(z_{1}^{2}+z_{5}^{2}\right)+\varepsilon\left(z_{0} z_{2}+z_{4} z_{6}\right)+\varepsilon \lambda\left(z_{2} z_{4}+z_{6} z_{0}\right)+\delta z_{3} z_{7}, \\
& J_{2}=\frac{1}{2}\left(z_{2}^{2}+z_{6}^{2}\right)+\varepsilon\left(z_{1} z_{3}+z_{5} z_{7}\right)+\varepsilon \lambda\left(z_{3} z_{5}+z_{7} z_{1}\right)+\delta z_{0} z_{4}, \\
& J_{3}=\frac{1}{2}\left(z_{3}^{2}+z_{7}^{2}\right)+\varepsilon\left(z_{2} z_{4}+z_{6} z_{8}\right)+\varepsilon \lambda\left(z_{4} z_{6}+z_{0} z_{2}\right)+\delta z_{5} z_{1} .
\end{aligned}
$$


This way of writing the equations is a more convenient starting point for investigating the transversality of the intersection $J_{k}=0$, because each $J_{k}$ is the sum of relatively few monomials. The actual calculations will not be given here, as we will treat a similar model below.

For our next model, we break up the eight homogeneous coordinates of $C P^{7}$ into two groups $z_{k}$, and $w_{k}, k=1,4$. We define transformations $U, V, \widetilde{V}$, and $W$ by

$$
\begin{array}{cc}
U\left(z_{k}\right)=z_{k+1}, & U\left(w_{k}\right)=w_{k+1}, \\
V\left(z_{k}\right)=\beta^{k} z_{k}, & V\left(w_{k}\right)=i \beta^{k} w_{k}, \\
\tilde{V}\left(z_{k}\right)=\beta^{k} z_{k}, & \tilde{V}\left(w_{k}\right)=i \beta^{-k} w_{k}, \\
W\left(z_{k}\right)=z_{k}, & W\left(w_{k}\right)=-w_{k},
\end{array}
$$

where now $\beta=\exp 2 \pi i / 4$.

Then $U, V$, and $W$ generate an abelian group $G$ with 32 elements; it is isomorphic to $Z_{4} \times Z_{4} \times Z_{2}$. U, $\widetilde{V}$, and $W$ generate a group of $G^{\prime}$ of 32 elements which is nonabelian since $U \tilde{V} U^{-1} \tilde{V}^{-1}=W$. ( $G^{\prime}$ has the irreducible two dimensional representation $U=i \sigma_{1}, \tilde{V}=i \sigma_{2}, W=-1$, so it can lead eventually to rank five groups in four dimensions.)

We now will write down a set of four quadrics invariant under $G$ and $\widetilde{G}$. We will write them this time only in the form which is analogous to (2.4). The quadrics are

$$
\begin{gathered}
L_{0}=\frac{1}{2}\left(z_{0}^{2}+z_{2}^{2}\right)+\varepsilon z_{1} z_{3}+\delta w_{0} w_{2}, \\
L_{1}=\frac{1}{2}\left(z_{1}^{2}+z_{3}^{2}\right)+\varepsilon z_{0} z_{2}+\delta w_{1} w_{3}, \\
L_{2}=\delta z_{0} z_{2}+\varepsilon^{\prime} w_{1} w_{3}+\frac{1}{2}\left(w_{0}^{2}+w_{2}^{2}\right), \\
L_{3}=\delta z_{1} z_{3}+\varepsilon^{\prime} w_{0} w_{2}+\frac{1}{2}\left(w_{1}^{2}+w_{3}^{2}\right) .
\end{gathered}
$$

To see that $G$ acts freely, it is again enough to check the elements of order two, which are $U^{2}, V^{2}, W, U^{2} V^{2}, U^{2} W, V^{2} W$, and $U^{2} V^{2} W$. The calculations are straightforward. To check that $G^{\prime}$ acts freely is no additional work, since $V^{2}=\tilde{V}^{2}$ and the elements of order two in $G^{\prime}$ are the same as those in $G$. Hence $K / G$ and $K / G^{\prime}$ are two new models with two generations. They again are an isomeric pair with a common universal covering space. Double covers of $K / G$ or of $K / G^{\prime}$ again give new four generation models.

We conclude this section by proving that the quadrics in (2.6) intersect transversely for generic $\varepsilon, \varepsilon^{\prime}$, and $\delta$. What must be shown is that the equations $L_{0}$ $=L_{1}=L_{2}=L_{3}=\omega=0$ have no solution in $C P^{7}$; here $\omega$ is the four form

$$
\omega=d L_{0} \wedge d L_{1} \wedge d L_{2} \wedge d L_{3} .
$$

The equation $w=0$ is a multitude of equations for the $z_{i}$ and $w_{j}$; it means that when $\omega$ is expanded as a sum of monomials, each monomial being quartic in $d z_{i}$ and $d w_{i}$, the coefficient of each such monomial vanishes. Vanishing of the coefficients of

$$
\begin{aligned}
d z_{0} \wedge d z_{1} \wedge d z_{2} \wedge d z_{3}, \quad d w_{0} \wedge d w_{1} \wedge d w_{2} \wedge d w_{3}, \\
d z_{0} \wedge d z_{2} \wedge d w_{0} \wedge d w_{2}, \quad d z_{0} \wedge d z_{2} \wedge d w_{1} \wedge d w_{3}, \\
d z_{1} \wedge d z_{3} \wedge d w_{0} \wedge d w_{2}, \quad \text { and } d z_{1} \wedge d z_{3} \wedge d w_{1} \wedge d w_{3}
\end{aligned}
$$


can be seen (after some algebra) to be equivalent to the following six equations:

$$
\begin{gathered}
\left(z_{0}^{2}-z_{2}^{2}\right)\left(z_{1}^{2}-z_{3}^{2}\right)=0, \\
\left(w_{0}^{2}-w_{2}^{2}\right)\left(w_{1}^{2}-w_{3}^{2}\right)=0, \\
\left(z_{0}^{2}-z_{2}^{2}\right)\left(w_{0}^{2}-w_{2}^{2}\right)=0, \\
\left(z_{0}^{2}-z_{2}^{2}\right)\left(w_{1}^{2}-w_{3}^{2}\right)=0, \\
\left(z_{1}^{2}-z_{3}^{2}\right)\left(w_{0}^{2}-w_{2}^{2}\right)=0, \\
\left(z_{1}^{2}-z_{3}^{2}\right)\left(w_{1}^{2}-w_{3}^{2}\right)=0 .
\end{gathered}
$$

These equations admit several branches of solutions. One branch is $z_{0}^{2}=z_{2}^{2}, z_{1}^{2}=z_{3}^{2}$, $w_{0}^{2}=w_{2}^{2}$. The other branches lead to an equivalent analysis, so we will focus on this one.

The vanishing of the coefficient of $d z_{0} \wedge d w_{0} \wedge d w_{1} \wedge d w_{3}$ now gives

$$
\left(w_{1}^{2}-w_{3}^{2}\right)\left(z_{0} w_{0}+\varepsilon \varepsilon^{\prime} z_{2} w_{2}-\delta^{2} z_{2} w_{2}\right)=0 .
$$

If we satisfy this with $w_{1}^{2}=w_{3}^{2}$, then, given also $z_{0}^{2}=z_{2}^{2}, z_{1}^{2}=z_{3}^{2} \ldots$, it is easily seen that for generical $\varepsilon, \varepsilon^{\prime}$, and $\delta$, the equations $L_{k}=0$ have no solution. Given that $z_{0}^{2}$ $=z_{2}^{2}, w_{0}^{2}=w_{2}^{2}$, the only other way to obey (2.8) (for generical $\varepsilon^{\prime}$ and $\delta$ ) is $z_{0}=z_{2}=0$ or $w_{0}=w_{2}=0$. One again easily finds that $L_{k}=0$ is (generically) incompatible with $z_{0}=z_{2}=0, z_{1}^{2}=z_{3}^{2}, w_{0}^{2}=w_{2}^{2}$, and also with $w_{0}=w_{2}=0, z_{0}^{2}=z_{2}^{2}, z_{1}^{2}=z_{3}^{2}$. This completes the proof that the quadrics $L_{k}=0$ intersect transversely.

\section{Group Actions with Fixed Points}

In Sect. II, we described the construction of several Calabi-Yau manifolds of $\chi=-4$ and $\chi=-8$ by taking a simply connected space $K$ and dividing by a discrete group $G$ that acts freely.

If $G$ does not act freely, the space $K / G$ has singularities. The singularities arise at points $x \in K$ that are left fixed by elements $g \in G$ (other than the identity). Under certain conditions, these singularities can be repaired ${ }^{3}$ in a suitable way, giving a manifold of vanishing first Chern class which thus admits a metric of SU(3) holonomy. Thus let $T$ be a connected submanifold of $K$ left fixed by some subgroup $G_{0}$ of $G$. Suppose that the points in $K$ close to but not on $T$ are not left fixed by any element of $G$. Then $G_{0}$ induces an action in the normal bundle $Q$ of $T$ in $K$. If this action has certain properties, the singularity of $K / G$ near $T$ can be repaired. This can happen under conditions to be described if $T$ is an isolated point or is a complex curve.

If $T$ is an isolated point, $p$, its normal bundle $Q$ has complex dimension three, and the action of $G_{0}$ on $Q$ is an embedding of $G_{0}$ in U(3). One case in which the singularity can be removed is that $G_{0}$ is $Z_{3}$ and $G_{0}$ transformations act on $Q$ as multiples of the identity (i.e. as elements of the $U(1)$ subgroup of $U(3)$ that commutes with $\mathrm{SU}(3)$ ). Then the singularity of $K / G$ near $p$ can be eliminated by removing $p$ and gluing in the three complex dimensional analogue [15] of the

3 This procedure is known as "blowing up" in the mathematics literature [14] 
Eguchi-Hanson space [16] (the -3 power of the Hopf bundle of $C P^{2}$, which admits a metric of SU(3) holonomy). As we show in the appendix, this space is asymptotically flat and topologically $S^{5} / Z_{3}$, so "the key fits the lock." Use of this procedure in a special case was sketched in [4].

The other relevant case arises if $T$ has complex dimension one. The simplest case is when $G_{0}$ is $Z_{2}$. The singularity of $K / G$ near $T$ can then be cured by removing $T$ and gluing in a copy of $T \times Z$ (or actually a suitable fiber bundle over $T$ with fiber $Z$ ) where $Z$ is the Eguchi-Hanson space. There are generalizations of this in which $G$ is not $Z_{2}$ but some discrete subgroup of SU(2); $Z$ must be replaced by a suitable generalization of the Eguchi-Hanson metric. We will not consider these generalizations here.

To illustrate these ideas, consider as in Sect. II the intersection $K$ of four quadrics $I_{k}$ in $C P^{7}$. Let $G$ be the $Z_{2}$ group generated by the diagonal matrix

$$
g=\left(\begin{array}{llllllll}
1 & & & & & & & \\
& 1 & & & & & & \\
& & 1 & & & & & \\
& & & 1 & & & & \\
& & & & 1 & & & \\
& & & & & 1 & & \\
& & & & & & -1 & \\
& & & & & & & -1
\end{array}\right)
$$

acting on eight homogeneous variables $z_{1}, z_{2}, \ldots, z_{8}$.

Take the $I_{k}$ to be any generical four quadrics that are invariant under $g$. Then $G$ does not act freely on $K$, since $g$ has fixed points. The fixed point set of $g$ in $C P^{7}$ is a copy of $C P^{5}\left(z_{7}=z_{8}=0\right)$ and a copy of $C P^{1}\left(z_{1}=\ldots=z_{6}=0\right)$. This $C P^{1}$ will generically not intersect $K$ (since four quadratic equations for $z_{7}$ and $z_{8}$ will have no solution). But the $C P^{5}$ that is left fixed by $g$ will intersect $K$ in a manifold $T$ of complex dimension one.

The Euler characteristic $\chi$ of $T$ is -32 , as may be computed by methods indicated in [4] (using the fact that $T$ is the intersection of four quadrics in $C P^{5}$ ).

Let us now calculate the Euler characteristic of the manifold $K$ made by removing $T$ from $K$, dividing by $G=Z_{2}$, and gluing in $T \times Z$. As $K$ has $\chi=-128$, $K-T$ has $\chi=-128-(-32)=-96$, so $(K-T) / Z_{2}$ has $\chi=-96 / 2=-48$. Gluing in $T \times Z$, we must add $\chi(T \times Z)=\chi(T) \chi(Z)=-32 \chi(Z)=-64$. So $K$ has $\chi=-48$ $+(-64)=-112$.

Having explained the strategy, we now turn to a more interesting example. We return to the last model of Sect. II. For convenience we rewrite the four quadrics:

$$
\begin{gathered}
L_{0}=\frac{1}{2}\left(z_{0}^{2}+z_{2}^{2}\right)+\varepsilon z_{1} z_{3}+\delta w_{0} w_{2}, \\
L_{1}=\frac{1}{2}\left(z_{1}^{2}+z_{3}^{2}\right)+\varepsilon z_{0} z_{2}+\delta w_{1} w_{3}, \\
L_{2}=\delta z_{0} z_{2}+\varepsilon^{\prime} w_{1} w_{3}+\frac{1}{2}\left(w_{0}^{2}+w_{2}^{2}\right), \\
L_{3}=\delta z_{1} z_{3}+\varepsilon^{\prime} w_{0} w_{2}+\frac{1}{2}\left(w_{1}^{2}+w_{3}^{2}\right) .
\end{gathered}
$$


As was explained in Sect. II, a non-abelian group $F$ of 32 elements, generated by $U, \widetilde{V}$, and $W$,

$$
\begin{array}{cc}
U\left(z_{k}\right)=z_{k+1}, & U\left(w_{k}\right)=w_{k+1}, \\
\tilde{V}\left(z_{k}\right)=\beta^{k} z_{k}, & \tilde{V}\left(w_{i}\right)=i \beta^{-k} w_{k}, \\
W\left(z_{i}\right)=z_{k}, & W\left(w_{k}\right)=-w_{k},
\end{array}
$$

acts freely on the space $K$ defined by $L_{k}=0$. (Here $\beta=\exp 2 \pi i / 4$.) Hence $K^{\prime}=K / F$ has $\chi=-4$.

Now consider the operation $Y$ defined by

$$
\begin{aligned}
& Y\left(z_{k}\right)=i^{-1 / 2} \beta^{k^{2} / 2} w_{k}, \\
& Y\left(w_{k}\right)=i^{1 / 2} \beta^{-k^{2} / 2} z_{k} .
\end{aligned}
$$

It is easy to check that $Y^{2}=1$, so $Y$ generates a $Z_{2}$ group $G$. It is easy to see that $Y$ is a symmetry of $K$ if $\varepsilon^{\prime}=i \varepsilon$. It is less obvious, but true, that $Y$ is a symmetry of $K / F$. [Proving this requires checking that the action of $Y$ on $K / F$ makes sense. In $K / F, x$ and $f x$ are equivalent for $x \in K$ and $f \in K$. The action of $Y$ on $K / F$ only makes sense if $Y(f x)$ is always equivalent to $Y(x)$. This means that for any $f \in F$ there must be $f^{\prime} \in F$ such that $Y(f x)=f^{\prime} Y(x)$. Since $x$ is arbitrary, it must be that for each $f \in F$ there is $f^{\prime} \in F$ with $Y f=f^{\prime} Y$. This can be shown with some effort. This step would fail if we used $V$ instead of $\tilde{V}$ in (3.3).]

Although $Y$ is a symmetry of $K / F$, it does not act freely on $K / F$. The fixed set of $Y$ in $K / F$ consists, however, of a subspace of complex dimension one, so the construction described above can be carried out.

In $C P^{7}$, the fixed set of $Y$ is two copies of $C P^{3}$, defined by $w_{0}=i^{1 / 2} \eta z_{0}, w_{1}=\eta z_{1}$, $w_{2}=-i^{1 / 2} \eta z_{2}, w_{3}=\eta z_{3}$, where $\eta=1$ or $\eta=-1$. If, say, $\eta=1$, the copy of $C P^{3}$ left fixed by $Y$ intersects $K$ (defined by $L_{k}=0$ ) on points obeying

$$
\begin{gathered}
\frac{1}{2}\left(z_{0}^{2}+z_{2}^{2}\right)+\varepsilon z_{1} z_{3}-i \delta z_{0} z_{2}=0, \\
\frac{1}{2}\left(z_{1}^{2}+z_{3}^{2}\right)+\varepsilon z_{0} z_{2}+\delta z_{1} z_{3}=0 .
\end{gathered}
$$

These two quadrics in the four homogeneous variables $z_{i}$ define a space $T_{1}$ of complex dimension one. By methods sketched in [4], it can be shown to have $\chi=0$ (which is the value of $\chi$ for any transverse intersection of two quadrics in $C P^{3}$ ). Setting $\eta=-1$ gives a distinct but isomorphic space $T_{2}$ of fixed points of $Y$ in $K$.

To analyze the fixed points of $Y$ in $K / F$ is trickier. A point $x$ in $K / F$ is left fixed by $Y$ if $Y x=f x$ for some $f$ in $F$. With some effort (surveying the possible choices of $f$ ), it can be shown that the fixed point set of $Y$ in $K / F$ is a space $T$ whose connected components $T_{i}$ are similar to $T_{1}$ and $T_{2}$ just considered. (They are defined by two quadrics in $C P^{3}$, modulo division by $F$.) Thus, $T$ has $\chi=0$.

Now we construct a new space $\tilde{K}$ by removing $T$ from $K / F$, dividing $K / F-T$ by $Y$, and gluing in a copy of $T \times Z$. As $K / F$ has $\chi=-4$ and $T$ has $\chi=0, K / F-T$ has $\chi=-4-0=-4 ;(K / F-T) / Z_{2}$ has $\chi=-2$; and $\tilde{K}=(K / F-T) / Z_{2}+T \times Z$ has $\chi=-2+\chi(T \times Z)=-2+\chi(T) \cdot \chi(Z)=-2$. Thus $\tilde{K}$ is the example - promised in the introduction - of a model of $\chi=-2$, and hence one generation of quarks and leptons. 
A relatively simple model with an odd number of generations can be obtained from $Y(5 ; 4,2)$. Consider the set of equations in $C P^{5}$ :

$$
E_{1}=\frac{1}{4} \sum_{i=1}^{3}\left(z_{i}^{4}-y_{i}^{4}\right)=0, \quad E_{2}=\sum_{i=1}^{3} \alpha_{i} z_{i} y_{i}=0
$$

with $\alpha_{i}$ arbitrary constants.

The normals to the two hypersurfaces defined by $E_{1}$ and $E_{2}$ are

$$
d E_{1}=\sum\left(z_{i}^{3} d z_{i}-y_{i}^{3} d y_{i}\right), \quad d E_{2}=\sum\left(\alpha_{i} z_{i} d y_{i}+\alpha_{i} y_{i} d z_{i}\right)
$$

and vanish nowhere on $C P^{6}$. The two form $d E_{1} \wedge d E_{2}$ vanishes if and only if $z_{i}^{3}$ $=\lambda \alpha_{i} y_{i}$ and $y_{i}^{3}=-\lambda \alpha_{i} z_{i}$ for some $\lambda$. The solutions of these equations are at

$$
z_{i}^{8}=-\lambda^{4} \alpha_{i}^{4}, \quad y_{i}^{8}=-\lambda^{4} \alpha_{i}^{4} .
$$

For generic $\alpha_{i}$, none of these points lie on the intersection of hypersurface $E_{1}$ $=E_{2}=0$, so the intersection is transverse.

Now consider the discrete $Z_{2}$ symmetry

$$
X\left(y_{i}\right)=z_{i}, \quad X\left(z_{i}\right)=y_{i} .
$$

The fixed point set in $C P^{5}$ is $z_{i}=y_{i}$ or $z_{i}=-y_{i} . E_{1}$ automatically vanishes there, so the fixed point set in the intersection consists of two disjoint components each of which is a quadric in $C P^{2}$, i.e. two $C P^{1}$ 's. Subtracting this set, dividing by $X$, and gluing back in the fixed point set times Eguchi-Hanson, one finds $\chi=\frac{1}{2}(-176-4)$ $+2 \cdot 2 \cdot 2=-82$. This gives 41 familes.

We now consider the construction of new four generation models with the intersection of two cubics in $C P^{5}$ as the starting point. Let

$$
E_{1}=\sum_{k=1}^{3}\left(z_{k}^{3}+w_{k}^{3}\right), \quad E_{2}=\sum_{k=1}^{3}\left(z_{k}^{3}-w_{k}^{3}\right) \alpha^{k},
$$

where $\alpha^{3}=1$. To check transversality of the intersection, note that the normals

$$
d E_{1}=3 \sum_{k=1}^{3}\left(z_{k}^{2} d z_{k}+w_{k}^{2} d w_{k}\right), \quad d E_{2}=3 \sum_{k=1}^{3}\left(z_{k}^{2} d z_{k}-w_{k}^{2} d w_{k}\right) \alpha^{k}
$$

vanish only if $w_{k}=0=z_{k}$. They are proportional if and only if all the $z_{k}, w_{k}$ but one vanish. $E_{i}=0$ then forces the remaining coordinate to vanish, so $d E_{1} \wedge d E_{2} \neq 0$ everywhere on the intersection. The $E_{i}$ are invariant under

$$
\begin{gathered}
V\left(z_{k}\right)=\alpha^{k} z_{k}, \\
V\left(w_{k}\right)=\alpha^{k} w_{k}, \\
S\left(z_{k}\right)=z_{k+1}, \\
S\left(w_{k}\right)=w_{k+1} .
\end{gathered}
$$

Fixed points of $S$ and $V$ in $C P^{5}$ lie on a $C P^{1}$, but the equations $E_{i}=0$ have no solution on this $C P^{1}$, so $S$ and $V$ act freely on the intersection. Now consider the $Z_{2}$ action

$$
X\left(z_{k}\right)=w_{k}, \quad X\left(w_{k}\right)=z_{k} .
$$


The equations $E_{i}=0$ are invariant under $X$. The fixed points lie on the $C P^{2} S$ $z_{k}= \pm w_{k}$. For either sign, one of the two equations vanishes identically. The fixed point set is a single cubic in $C P^{2}$ which is isomorphic to a torus and has zero Euler character. This means that if we divide $Y(5 ; 3,3)$ by the group $G$ generated by $V, S$, and $X$, the resulting Euler character will be $\chi=\frac{\chi_{0}}{n(G)}=-\frac{144}{18}=-8$. Equations (3.10) are also invariant under a non-abelian group with twelve elements. This group is generated by $X, S$, and

$$
\tilde{V}\left(z_{k}\right)=\alpha^{k} z_{k}, \quad \tilde{V}\left(w_{k}\right)=\alpha^{-k} w_{k} .
$$

The fixed point set is again a torus, and this leads to another four generation model.

\section{Holomorphic Three Forms}

It is well known in the mathematics literature that the vanishing of the first Chern class is equivalent, in $n$ complex dimensions, to the existence of an everywhere nonsingular and non-zero holomorphic $n$ form. ${ }^{4}$ This equivalence is very useful for finding Calabi-Yau manifolds. In this section we will explain this equivalence in a manner accessible to physicists and describe how these $n$ forms can be explicitly constructed.

Recall that, on a complex manifold with a Kahler metric and associated connection, a representative of the first Chern class $\left(c_{1}\right)$ is the curl of the U(1) part of the connection. Locally, this may be written

$$
\begin{gathered}
R=d A, \\
A_{\bar{b}}=-i \Gamma_{\bar{b}}=\left(A_{b}\right)^{*}, \\
\Gamma_{\bar{b}} \equiv \Gamma_{\bar{b}}^{\bar{c}}=\frac{1}{2} \partial_{\bar{b}} \ln \operatorname{det} g .
\end{gathered}
$$

$R$ is also known as the Ricci form, as it is just the complex structure times the Ricci tensor. If $R$ is not in the trivial cohomology class (i.e. $A$ cannot be globally defined), there is a topological obstruction to obtaining a Ricci flat Kahler metric. It was conjectured by Calabi and proved by Yau that if $R$ is topologically trivial, i.e. $c_{1}=0$, then there does exist a Ricci flat Kahler metric. Ricci flatness imples that $\Gamma_{\bar{b}}$ vanishes (in some coordinate system) and that the holonomy group is $\mathrm{SU}(n)$.

It is easy to see that $\mathrm{SU}(n)$ holonomy implies the existence of a holomorphic $n$ form. For notational simplicity we hereafter consider only $n=3$. A $(3,0)$ form transforms as a singlet under the $\mathrm{SU}(3)$ part of the connection, but is charged with respect to the $U(1)$ part of the connection. If the $U(1)$ connection is topologically trivial (and vanishes for the Ricci flat metric) then a holomorphic three form always exists. For the special case of a Ricci flat metric, this three form can be constructed from the positive chirality covariantly constant spinors

$$
\omega=\zeta^{T} \gamma_{a b c} \zeta_{+} d z^{a} d z^{b} d z^{c} .
$$

4 A harmonic form of type $(k, 0)$ is called a holomorphic $k$ form 
To show that the existence of a holomorphic three form implies $c_{1}=0$, choose an arbitrary Kahler metric $g_{a \bar{b}}$. Then the associated Ricci form can be written

$$
R=d V, \text { where } \quad V_{\bar{d}}=i \partial_{\bar{d}} \ln \left(\omega_{a b c} \omega_{\bar{a} \bar{b} \bar{c}} g^{a \bar{a}} g^{b \bar{b}} g^{c \bar{c}}\right)=\left(V_{d}\right)^{*}
$$

The argument of the logarithm, which is proportional to $\operatorname{det}^{-1 / 2} \mathrm{~g}$, is the norm of $\omega$. If $\omega$ is everywhere non-singular, then $V$ is globally well defined, $R$ is in the trivial cohomology class and $c_{1}=0$. Similar arguments apply for $n \neq 3$.

We now describe how $\omega$ is constructed in simple cases. Consider a hypersurface in $C P^{4}$,

$$
\sum_{i=1}^{5} z_{i}^{n}=0
$$

where $z_{i}$ are homogeneous coordinates. It is convenient to introduce inhomogeneous coordinates $y_{k}=z_{k} / z_{5}, k=1, \ldots, 4$. The equation becomes

$$
1+f\left(y_{k}\right)=0 \quad \text { with } \quad f\left(y_{k}\right)=\sum_{i=1}^{4} y_{k}^{n} .
$$

Define a holomorphic three form $\omega$ by

$$
\omega=d y_{1} \wedge d y_{2} \wedge d y_{3} / y_{4}^{n-1} .
$$

We need to check that it is non-singular. $y_{4}=0$ appears to be a singular point. However $\omega$ may be rewritten as

$$
\omega=-d y_{2} \wedge d y_{3} \wedge d y_{4} / y_{4}^{n-1},
$$

using $d f(y)=0$. No difficulty arises unless all the $y_{k}$ 's vanish, a point which is not on the hypersurface (4.5). We must also investigate the point where one or more of the $y_{i}$, say $y_{1}$, becomes infinite, which is equivalent to $z_{5} \rightarrow 0$. To do so we change variables,

$$
x_{i}=z_{i} / z_{1}=y_{i} / y_{1} \text {. }
$$

In terms of the $x_{i}$,

$$
\omega=-x_{5}^{n-5} d x_{2} \wedge d x_{3} \wedge d x_{5} / x_{4}^{n-1} .
$$

So we see $\omega$ is nonsingular and non-zero at the point $z_{5}=0=x_{5}$ if and only if $n=5$. Thus a quintic hypersurface in $C P^{4}$ has $c_{1}=0$.

This construction is readily generalized. For a generic quintic polynomial in $C P^{4}$ which in inhomogeneous coordinates takes the form

$$
1+f\left(y_{i}\right)=0
$$

the holomorphic three form

$$
\omega=d y_{1} \wedge d y_{2} \wedge d y_{3} /\left(\partial f / \partial y_{4}\right)
$$

is everywhere non-singular and non-zero. One may also consider a system of $k$ equations in $C P^{k+3}$ :

$$
1+f_{a}\left(y_{i}\right)=0, \quad i=1, \ldots, k+3, \quad a=1, \ldots, k .
$$


The appropriate three form is

$$
\omega=d y_{k+1} \wedge d y_{k+2} \wedge d y_{k+3} /\left(\operatorname{det} \frac{\partial f_{a}}{\partial y_{b}}\right), \quad a, b=1, \ldots, k .
$$

Potential difficulties for some $y_{\mathrm{c}}=0$ can be seen not to exist by rewriting $\omega$,

$\omega=d y_{c} \wedge d y_{k+2} \wedge d y_{k+3} /\left(\operatorname{det} \frac{\partial f_{a}}{\partial y_{b}}\right), \quad a=1, \ldots, k, \quad b=1, \ldots, c, \ldots, k+1$.

Restrictions on the degrees of the polynomials arise from inspection of the region $z_{k+4}=0$. To investigate this region, one uses variables

$$
x_{i}=y_{i} / y_{1}=z_{i} / z_{1}
$$

in terms of which

$$
\omega=d x_{k+2} \wedge d x_{k+3} \wedge d x_{k+4} \times \sum_{k+4} D_{j}-k-4 / \operatorname{det} \frac{\partial f_{a}(x)}{\partial x_{b}} .
$$

Thus $c_{1}=0$ if $\sum^{k} D_{j}=k+4$. This reproduces the five algebraic varieties discussed in [4]. In the following section generalizations of this technique will be used to find new Calabi-Yau manifolds.

\section{Branched Coverings}

Consider the equation

$$
z_{0}^{M}+\sum_{i=1}^{n+1} z_{i}^{M K}=0
$$

where the identification $z_{i} \simeq \lambda z_{i} ; z_{0} \simeq \lambda^{K} z_{0}$ is made. For each point $\left(z_{1}, \ldots, z_{n+1}\right)$ in $C P^{n}$ not on the hypersurface $V$ defined by $\sum z_{i}^{M K}=0$ there are $M$ values of $z_{0}$. On $V$ there is only the solution $z_{0}=0$. Thus we describe this manifold as a branched cover of $C P^{n}$ branched $M$ times over the hypersurface $V$.

To illustrate the concept of a branched covering, we consider a simple example. The torus can be described as a branched cover of $C P^{1}$. Consider the equation

$$
z_{0}^{2}+\sum_{i=1}^{2} z_{i}^{4}=0
$$

This can be identified as the torus by computing the Euler number. If $V$ is the surface $\Sigma z_{i}^{4}=0$, then $\chi=2\left[\chi\left(C P^{1}\right)-\chi(V)\right]+\chi(V)$, since $C P^{1}-V$ is covered twice and $V$ is covered once. $V$ is simply the four points $z_{1}=\alpha_{k} z_{2}$, where $\alpha_{k}$ is a fourth root of minus one. $V$ thus has $\chi=2$, while $C P^{1}$ has $\chi=2$. Thus the surface (5.2) has $\chi=0$ and describes a torus.

We now use this construction to generate new manifolds with SU(3) holonomy. There are two branched covers of $C P^{3}$ with $c_{1}=0$. We consider equations of the form

$$
z_{0}^{N}+\sum_{i=1}^{4} z_{i}^{M N}=0, \quad z_{0} \simeq \lambda^{M} z_{0}, \quad z_{i} \simeq \lambda z_{i} .
$$



This describes an $n$-fold cover of $C P^{3}$, branched over the locus $\sum_{i=1}^{4} z_{i}^{M N}=0$. It can
be alternatively written:

$$
1+f(y)=0
$$

where

$$
f(y)=\sum_{i=1}^{4} y_{i}^{M N}, \quad y_{i}=z_{i} / z_{0}^{1 / M}
$$

The (potentially) holomorphic three form is

$$
\omega=d y_{1} \wedge d y_{2} \wedge d y_{3} /\left(\partial f / \partial y_{4}\right) .
$$

Finiteness at $z_{0}=0$ is checked by a change of variables. We find the condition

$$
N M=4+M \text {. }
$$

This has the two solutions $N=2, M=4$ and $N=3, M=2$. Thus the two equations,

$$
\begin{aligned}
& z_{0}^{2}+\sum_{i=1}^{4} z_{i}^{8}=0, \\
& z_{0}^{3}+\sum_{i=1}^{4} z_{i}^{6}=0,
\end{aligned}
$$

give manifolds with $c_{1}=0$. If $V$ is the hypersurface where branching occurs, then these manifolds have $\chi=N \chi\left(C P^{3}\right)+(1-N) \chi(V) \cdot \chi(V)$ can be calculated from the Chern character as indicated in [4]. (5.6a) has $\chi=-296$ while (5.6b) has $\chi=-204$.

Similarly one may consider intersections of $k N$-branched coverings of $C P^{2+k}$, but none of them have $c_{1} \neq 0$ for $k>1$. More interesting is to consider the branched covering of a complete intersection of hypersurfaces. Consider the equations

(i) $z_{0}^{D_{a}}+\sum_{j=1}^{3+k+l} z_{j}^{M D_{a}}=0, \quad a=1, \ldots, k$,

(ii) $\sum_{j=1}^{3+k+l} z_{j}^{d_{b}}=0, \quad b=1, \ldots, l, \quad z_{0} \simeq \lambda^{M} z_{0}, \quad z_{i} \simeq \lambda z_{i}$.

These have the following interpretation. For each point in $C P^{2+k+l}$ obeying (ii), there are $D_{a}$ values of $z_{0}$, unless $\sum z_{j}^{M D_{a}}=0$, in which case there is only $z_{0}=0$. Hence the manifold is a branched cover of the hypersurface defined by (ii), the branch locus being the intersection of that hypersurface with the hypersurface defined by $\sum z_{j}^{M D_{a}}=0$. The condition for $c_{1}=0$ is again determined by constructing the holomorphic three form. We find $3+k+l+M=\sum_{a=1}^{k} D_{a}+\sum_{b=1}^{l} d_{b}$. There are three new solutions:

(1) $z_{0}^{2}+\sum_{i=1}^{5} z_{i}^{4}=0, \quad \sum_{i=1}^{5} z_{i}^{3}=0, \quad \chi=-156$,

(2) $z_{0}^{2}+\sum_{i=1}^{4} z_{i}^{6}=0, \quad \sum_{i=1}^{6} z_{i}^{2}=0, \quad \sum_{i=1}^{6} a_{i} z_{i}^{2}=0, \quad \chi=-176$

(3) $z_{0}^{2}+\sum_{i=1}^{5} z_{i}^{6}=0, \quad \sum_{i=1}^{5} z_{i}^{2}=0, \quad \chi=-256$. 
Another possibility is to consider arbitrary weighted homogeneous spaces

$$
z_{i} \simeq \lambda^{M_{i}} z_{i} .
$$

In general these spaces are singular because the transformation $z_{i} \simeq \lambda^{M_{i}} z_{i}$ has fixed points when $\lambda^{M_{i}}=1$. One must be sure that the hypersurfaces defined by the equations:

$$
\sum_{i=1}^{5} z_{i}^{D} \prod_{j \neq i} M_{j}
$$

avoids these singularities. Modulo, this singularity problem, the holomorphic three form is globally defined if

$$
\sum_{i=1}^{5} M_{i}=D \prod_{j \neq i} M_{j} .
$$

The singularities are avoided if the $M_{j}$ are relatively prime. Only one new manifold is found this way:

$$
\begin{gathered}
z_{1}^{5}+z_{2}^{2}+z_{3}^{10}+z_{4}^{10}+z_{5}^{10}=0 \\
z_{1} \simeq \lambda^{2} z_{1}, \quad z_{2} \simeq \lambda^{5} z_{2}, \\
\left(z_{3}, z_{4}, z_{5}\right) \simeq \lambda\left(z_{3}, z_{4}, z_{5}\right) .
\end{gathered}
$$

Note that the singularity at $\lambda=-1, z_{2}, \ldots, z_{5}=0, z_{1} \neq 0$ in the weighted homogeneous space is avoided by the hypersurface because Eq. (5.12) implies that if $z_{2}, \ldots, z_{5}$ vanish $z_{1}$ must as well. The singularity for nonzero $z_{2}$ is similarly avoided.

Clearly the constructions presented here have many generalizations. A noticeable feature is that more complicated constructions tend to produce more complicated manifolds with larger $|\chi|$.

\section{VI. $\mathscr{C} \mathscr{P}$ Violation}

In this section we will discuss the question of $\mathscr{C} \mathscr{P}$ conservation in Calabi-Yau manifolds.

$N=1$ supergravity in ten dimensions is not invariant under parity. Thus, if this theory compactifies to $M^{4} \times K$, where $M^{4}$ is four dimensional Minkowski space and $K$ is a complex manifold of complex dimension three, there will be no invariance under the usual parity transformation $P$ of $M^{4}(P$ being $t \rightarrow t, \mathbf{x} \rightarrow-\mathbf{x})$.

However, suppose we find an isometry $\hat{\mathscr{C}}$ of $K$ which reverses the orientation of $K$. Then $\hat{\mathscr{C}} \mathscr{P}$ preserves the orientation of $M^{4} \times K$, and is a symmetry of the theory. In almost all relevant cases, $\hat{\mathscr{C}}$ has the properties of standard charge conjugation, and $\hat{\mathscr{C}} \mathscr{P}$ can be called $\mathscr{C} \mathscr{P}$.

What orientation reversing symmetry might a manifold of $\mathrm{SU}(3)$ holonomy have? A transformation that preserves the complex structure always preserves the orientation. However a so-called antiholomorphic transformation that exchanges the independent complex variables $z_{i}, i=1, \ldots, 3$ with their complex conjugates always reverses the orientation. (The reason for this is that if $z_{i}=u_{i}+i v_{i}, u_{i}$ and $v_{i}$ being real, then complex conjugation is $u_{i} \rightarrow u_{i}, v_{i} \rightarrow-v_{i}$; as there are an odd 
number of $v_{i}$, this reverses the orientation.) An orientation reversing isometry of a manifold whose holonomy group is SU(3) (rather than a subgroup of SU(3)) is always an antiholomorphic transformation. ${ }^{5}$ Such a transformation deserves to be called $\mathscr{C}$ since it reverses the 27 and $\overline{2} \overline{7}$ of $E_{6}$ in the construction of [4]. The combined operation $\left(t, \mathbf{x}, z_{i}\right) \rightarrow\left(t,-\mathbf{x}, z_{i}^{*}\right)$ deserves to be called $\mathscr{C} \mathscr{P}$.

When does a manifold of SU(3) holonomy admit an antiholomorphic transformation? Consider the example of a quintic hypersurface in $C P^{4}$. The simplest quintic is

$$
\sum_{i=1}^{5} z_{i}^{5}=0
$$

The transformation $z_{i} \rightarrow z_{i}^{*}$ defines a $Z_{2}$ action on the hypersurface. Equally good (in that it leads to a metric of $\mathrm{SU}(3)$ holonomy) would be a more general quintic such as

$$
\sum_{i=1}^{5} z_{i}^{5}+\varepsilon z_{1} z_{2} z_{3} z_{4} z_{5}=0
$$

If $\varepsilon$ is real there is still a symmetry $z_{i} \rightarrow z_{i}^{*}$, but if $\varepsilon$ is complex this symmetry is absent, since $z_{i}$ being on the hypersurface does not imply that $z_{i}^{*}$ is on the hypersurface.

There are several points of view one can take about this. At tree level any (non-singular) quintic defines a candidate vacuum state. If this degeneracy is not lifted, then one can allege that the generic quintic is $\mathscr{C} \mathscr{P}$ violating. If the degeneracy is lifted, then the question of $\mathscr{C} \mathscr{P}$ conservation depends entirely on how it is lifted. It would be very interesting to find examples of manifolds of SU(3) holonomy that violate $\mathscr{C} \mathscr{P}$ regardless of what Ricci flat metric ic chosen, but we do not know of such examples.

One might at first think that $\mathscr{C P}$ violation in the defining equation of our manifold would tend to be stronger than what is observed in nature, but this is not so; in the Kobayashi-Maskawa model of $\mathscr{C} \mathscr{P}$ violation, maximal $\mathscr{C} \mathscr{P}$ violating angles are quite acceptable.

\section{Yukawa Couplings}

In this section we will discuss the extent to which Yukawa couplings can be computed from topological invariants or invariants of the topology plus complex structure.

One particularly interesting result we will find is that whether or not a given Yukawa coupling vanishes at tree level can be predicted on topological grounds without having so solve any equations. This is likely to be important because many problems about quark and lepton masses and mixing (and about other problems such as proton decay) may be related to vanishing of tree level Yukawa couplings. As for non-zero Yukawa couplings, because of normalization factors involving the

5 This is so because it must map the covariantly constant positive chirality spinor $\zeta$ into a negative chirality covariantly constant spinor - which must be $\zeta^{*}$ if the holonomy is precisely SU(3). A transformation that exchanges $\zeta$ and $\zeta^{*}$ precisely changes the sign of the complex structure 
metric that will appear later, topological arguments only give relations among them.

Four dimensional Yukawa couplings arise from cubic (or higher) terms in the ten dimensional action. For example, the ten dimensional coupling

$$
L_{I}=\int d^{10} w \sqrt{-g} \bar{\psi}_{A}(w) \gamma^{m} A_{m B}(w) \psi_{C}(w) f^{A B C},
$$

where $A, B, C$ are $E_{8} \times E_{8}$ indices, can be expanded in harmonics on the internal manifold $K$ (suppressing gauge indices)

$$
\begin{gathered}
\psi(w)=\sum_{i} \psi^{i}(x) \otimes \psi^{i}(y), \\
A_{m}(w)=\sum_{i} A_{m}^{i}(x) \phi^{i}(y)+\sum_{j} \phi^{j}(x) A_{m}^{j}(y), \\
\gamma^{m} D_{m} \psi^{i}(y)=\lambda^{i} \psi^{i}(y), \\
D^{2} A_{m}^{i}(y)-R_{m}^{n} A_{n}^{i}(y)+2 F_{m}^{n} A_{n}^{i}(y)=\lambda^{i} A_{m}^{i}(y), \quad D^{2} \phi^{j}(y)=\lambda^{j} \phi^{j}(y),
\end{gathered}
$$

where $x$ parameterizes spacetime, $y$ parameterizes $K$ and $D$ is the gauge covariant derivative on $K$. Each mode on $K$ corresponds to a four dimensional field with mass proportional to $\lambda^{i}$. In order that the four dimensional kinetic terms are properly normalized, the internal harmonics should be normalized so that

$$
\int_{K} d^{6} y \sqrt{g} \Phi^{* i}(y) \Phi^{j}(y)=\delta^{i j} .
$$

Yukawa couplings among the light particles involve zero modes on $K$. Plugging (2) into (1) we find terms of the form:

$$
L_{I}=\int_{K} d^{6} y \sqrt{g} \psi^{0 \dagger}(y) A^{0}(y) \psi^{0}(y) \int d^{4} x \sqrt{-g} \bar{\psi}^{0}(x) \phi^{0}(x) \psi^{0}(x) .
$$

The effective Yukawa coupling is thus the overlap integral of the zero mode wave functions:

$$
g=\int d^{6} y \sqrt{g} \psi^{0 \dagger}(y) A^{0} \psi^{0}(y) .
$$

$A_{m}^{a}$ is a one form with an extra gauge index $a$. For charged matter fields, $a$ labels the 3 or $\overline{3}$ of $S U(3)$ (coming from $(27,3) \oplus(\overline{2} \overline{7}, \overline{3})$ in the adjoint representation of $E_{8}$ ). The 3 and $\overline{3}$ label the holomorphic tangent and cotangent bundles $T$ and $\bar{T}$ respectively, so $A_{m}^{a}$ is a one form with values in $T$ or $\bar{T}$. It will turn out that gauge field zero modes are realted to elements of $H^{1}(T)$ and $H^{1}(\bar{T})$, the first $\bar{\partial}$ cohomology group with values in $T$ or $\bar{T}$.

For one forms with values in $T$ the1e is a natural exterior derivative given by:

$$
\bar{\partial} A=\partial_{\bar{n}} A_{\bar{m}}^{a} d z^{\bar{n}} \wedge d z^{\bar{m}} \frac{\partial}{d z^{a}} .
$$

The letters $a, b, c$ will be used for tangent space indices. Note that under a holomorphic change of frames:

$$
\begin{gathered}
A_{\bar{m}}^{\prime a}=\Lambda_{b}^{a}(z) A_{\bar{m}}^{b}, \\
\left(\frac{\partial}{\partial z^{a}}\right)^{\prime}=\left(\Lambda^{-1}(z)\right)_{a}^{T b} \frac{\partial}{\partial z^{b}},
\end{gathered}
$$


(7.6) is invariant. The adjoint of $\bar{\partial}$ can also be defined for a given Kahler metric. One finds:

$$
\left(\bar{\partial}^{*} A\right)^{b}=\nabla^{\bar{m}} A_{\bar{m}}^{b},
$$

where $\nabla^{m}$ is the usual covariant derivative. We now observe the following remarkable fact: if the holomorphic tangent indices are identified with $\mathrm{SU}(3)$ gauge indices in the 3 representation, and the background gauge field is given by $\left(A_{B m}\right)_{a}^{b}$ $=\Gamma_{m a}^{b} ;\left(\mathrm{A}_{B \bar{m}}\right)_{\bar{a}}^{\bar{b}}=\Gamma_{\bar{m} \bar{a}}^{\bar{b}}$, Eqs. (7.6) and (7.8) become precisely the equations for the gauge covariant curl and divergence of $A$. It further follows that $A$ is harmonic,

$$
\left(\bar{\partial}^{*} \bar{\partial}+\bar{\partial}^{*}\right) A^{b}=0 \text {. }
$$

Equation (7.9) is identical to Eq. (7.2) when $\left(A_{B m}\right)_{b}^{a}=\Gamma_{m b}^{a}$. Thus zero modes of the Yang-Mills field in the 3 representation are given by harmonic elements of $H^{1}(T)$.

Zero modes of the Dirac equation are now constructed using supersymmetry as:

$$
\psi^{a}=A_{\bar{m}}^{a} \gamma^{\bar{m}} \zeta_{+},
$$

where $\gamma \zeta_{ \pm}= \pm \zeta_{ \pm}, \zeta_{ \pm}^{*}=\zeta_{\mp}$ and $\gamma=i \gamma_{5}, \ldots, \gamma_{10}$. In verifying that $\psi^{a}$ is a zero mode, the relation $\gamma^{m} \zeta_{+}=0$ is useful. $\zeta_{-}$can not be used in (7.10), since $\gamma^{\bar{m}} \zeta_{-}=0$. Thus harmonic elements of $H^{1}(T)$ lead only to left-handed fermions in the 3 of SU(3). Since the theory contains fermions in the $(27,3)$ of $E_{6} \times \operatorname{SU}(3)$, these give rise to 27's of $E_{6}$ at low energies.

For the special case of a Ricci flat metric on $K$, there is a map from harmonic elements of $H^{1}(T)$ to harmonic $(2,1)$ forms. This is given by the holomorphic and (for the Ricci flat case) covariantly constant three form,

$$
G_{\bar{m} n p}=A_{\bar{m}}^{a} \omega_{\text {anp }},
$$

$\left(\bar{\partial}+\bar{\partial}^{*}\right)^{2} A=0$ immediately implies $\left(\bar{\partial}+\bar{\partial}^{*}\right)^{2} G=0$. On a Kahler manifold $\left(\bar{\partial}+\bar{\partial}^{*}\right)^{2}$ $=\left(\partial+\partial^{*}\right)^{2}=\frac{1}{2}\left(d+d^{*}\right)^{2}$, so $G$ is harmonic. This tells us that the number of families is $\operatorname{dim} H^{2,1}$.

Antifamilies are associated with elements of $H^{1}(\bar{T})$, where $\bar{T}$ is the holomorphic cotangent bundle. In this case the $\bar{\partial}$ harmonic elements obey:

$$
(\bar{\partial} A)_{a}=0=\left(\bar{\partial}^{*} A\right)_{a} .
$$

Zero modes of the Yang-Mills field in the $\overline{3}$ are given by

$$
\left(\left(\bar{\partial}^{*} \bar{\partial}+\overline{\partial \partial}^{*}\right) A\right)_{a}=0 .
$$

One obvious solution of (16), which exists on all Kahler manifolds, is $A_{\bar{m} a}=e_{\bar{m} a}$. Superpartners of $A_{\bar{m} a}$ will be left-handed fermions in the $\overline{3}$ representation.

Harmonic elements of $H^{1}(\bar{T})$ can be related to harmonic $(1,1)$ forms:

$$
V_{m \bar{n}}=-i A_{\bar{n} a} e_{m}^{a}
$$

so $\operatorname{dim} H^{1}(\bar{T})=b_{11}$. Since $\frac{\chi}{2}=b_{21}-b_{11}$ on a manifold of SU(3) holonomy, we regain the result that the net number of families is half the Euler character.

We now discuss Yukawa couplings among the four dimensional fields associated with these zero modes as given by the wave function overlap integral 
(7.5). There will be no cubic couplings between familes and antifamilies as there is no SU(3) invariant way to combine two elements of $H^{1}(T)$ with one of $H^{1}(\bar{T})$ or two elements of $H^{1}(\bar{T})$ with one of $H^{1}(T)$ (i.e. there is no singlet in $3 \otimes 3 \otimes \overline{3}$ ).

Cubic interactions between $\overline{2} \overline{7}$ 's are given by

$$
L_{i j k}=\bar{g}_{i j k} \int d^{4} x \sqrt{-g} \bar{\psi}_{i}^{\bar{A}} \gamma^{\bar{m}} A_{\bar{m} j}^{\bar{B}} \psi_{k}^{\bar{c}} d_{\bar{A} \bar{B} \bar{C}},
$$

where

$$
\bar{g}_{i j k}=\int_{K} d^{6} y \sqrt{g} \psi_{a i}^{\dagger} \gamma^{\bar{m}} A_{\bar{m} b j} \psi_{c k} \varepsilon^{a b c} .
$$

$\bar{A}, \bar{B}, \bar{C}$ are $E_{6}$ indices in the $\overline{2} \overline{7}$ representation, $d_{\bar{A} \bar{B} \bar{C}}$ is the symmetric cubic invariant in the $\overline{2} \overline{7}, a, b, c$ are $\mathrm{SU}(3)$ (or tangent space) indices, and $\psi_{a i}$ is the $i^{\text {th }}$ zero mode wave function in the $\overline{3}$ representation. Using the formula for the zero modes in terms of elements of $H^{1}(\bar{T})$, we find

$$
\bar{g}_{i j k}=\int d^{6} y \sqrt{g} \zeta_{+}^{T} \gamma^{\bar{m}} A_{\bar{m} a i} \gamma^{\bar{n}} A_{\bar{n} b j} A_{\bar{p} c k} \gamma^{\bar{p}} \zeta_{+} \varepsilon^{a b c} .
$$

Using the fact that

$$
\omega_{m n p}=\zeta_{+}^{T} \gamma_{m} \gamma_{n} \gamma_{p} \zeta_{+},
$$

we obtain

$$
\begin{aligned}
\bar{g}_{i j k} & =\int d^{6} y \sqrt{g} \omega^{\bar{m} \bar{n} \bar{p}} A_{\bar{m} a i} A_{\bar{n} b j} A_{\bar{p} c k} \varepsilon^{a b c} \\
& =\int_{K} \omega \wedge A_{a i} \wedge A_{b j} \wedge A_{c k} \varepsilon^{a b c},
\end{aligned}
$$

where $A_{a i}=A_{\bar{m} a i} d z^{\bar{m}}$. Note that this expression is symmetric in $i j k$, as required by supersymmetry. Now for the special case of a Ricci flat metric, covariant constancy of $\omega$ implies $\omega_{m n p}=\varepsilon_{m n p}$. This enables us to rewrite (7.18) in terms of the two form $V_{i}$ $=A_{\bar{m} a i} e_{n}^{a} d z^{\bar{m}} \wedge d z^{n}$ as:

$$
\bar{g}_{i j k}=\int_{K} V_{i} \wedge V_{j} \wedge V_{k} .
$$

The integrands of (7.18) and (7.19) do not in general agree for an arbitrary hermitian metric. (Being topological invariants, however, the integrals will agree.) The latter expression has the advantage that it does not in any way involve the complex structure on $K$, and can be defined for an arbitrary Riemannian metric. If the $V_{i}$ are normalized topologically (to represent integral cohomology classes), then the integral in (7.19) is determined purely topologically, in terms of the cohomology ring of the manifold $K$. This means (7.19) is invariant under $\delta V_{i}=d \lambda_{i}$ for any $\lambda_{i}$. In particular, there is no need to know the metric of $K$ or to solve the Dirac equation for the $V_{i}$, as any $V_{i}$ in the right cohomology class will give the same integral. In terms of homology, (7.19) counts the number of points of intersection of the 2 complex dimensional hypersurfaces associated with $V_{i}, V_{j}$, and $V_{k}$.

Actually, we want to normalize the $V_{i}$ not topologically but rather according to (7.3). As this normalization condition cannot be worked out topologically but requires knowledge of the metric and the wave functions, topological considerations suffice to give only certain relations among Yukawa couplings. Of course, whether $\bar{g}_{i j k}$ vanishes for certain $i, j$, and $k$ is purely a topological question. 
One can similarly compute the Yukawa couplings among families. They are given by a formula analogous to (7.18):

$$
g_{i j k}=\int_{\mathbf{K}} \omega \wedge A_{i}^{a} \wedge A_{j}^{b} \wedge A_{k}^{c} \varepsilon_{a b c}
$$

where $A_{i}^{a}=A_{\bar{m} i}^{a} d z^{\bar{m}}$ is the $i^{\text {th }}$ zero mode associated with the 3 representation. ${ }^{6}$ Equation (7.20) depends on the complex structure of $K$ (via $\omega$ ), but not on the metric tensor of $K$. Also, (7.20) can be evaluated without knowing the precise form of the $A_{i}^{a}$, only their cohomology class in $H^{1}(T)$. Again, the proper normalization of the $A_{i}^{a}$ cannot be determined topologically, so knowledge of the topology (and complex structure) gives only certain relations among Yukawa couplings.

\section{Appendix}

The form of the asymptotically flat, spherically symmetric metrics of SU( $n)$ holonomy was discovered by Calabi and discussed by Freedman and Gibbons. Properties of the two and three complex dimensional cases are important to our results and we describe them here.

The line element giving $\mathrm{SU}(n)$ holonomy in $n$ dimensions is

$$
d s^{2}=\left(1+x^{-n}\right)^{\frac{1}{n}} d z^{k} d \bar{z}^{k}-\frac{1}{x^{2}}\left(x^{n}+1\right)^{\frac{1}{n}-1} \bar{z}_{k} d z^{k} z_{j} d \bar{z}^{j},
$$

where $x=\bar{z}_{k} z_{k}$. As $x \rightarrow \infty$, the metric is flat, but near $x=0$, it appears singular. To investigate the origin we define new variables,

$$
y_{i}=z_{i} / z_{n}, \quad i=1, \ldots, n-1, \quad y=z_{n} .
$$

The origin $x=0$ is then $y=0, y_{i}$ finite. We have near $y=0$,

$$
\begin{aligned}
d s^{2}= & |y|^{2 n-2}\left(1+y_{i} \bar{y}_{i}\right)^{n} d y d \bar{y} \\
& +|y|^{2 n-2}\left(1+y_{i} \bar{y}_{i}\right)^{n-1}\left(\bar{y} d y y_{i} d \bar{y}^{i}+y d \bar{y} \bar{y}_{i} d y^{i}\right) \\
& +\left(1+y_{i} \bar{y}_{i}\right)^{-1} d y^{i} d \bar{y}^{i} \\
& -\left(1+y_{i} \bar{y}_{i}\right)^{-2}\left(y_{i} d \bar{y}^{i} \bar{y}_{j} d y^{j}\right)+0(y) .
\end{aligned}
$$

If we define a new variable $w=y^{n}$, the metric appears regular at $w=0$. However, as the phase of $w$ goes from 0 to $2 \pi$, the phase of $y$ goes only from 0 to $2 \pi / n$. Thus the metric appears regular if we identify $y$ with $e^{2 \pi i / n} y$. Approaching the origin from other directions, one finds that it is regular there if the identification $z_{k} \simeq e^{2 \pi i / n} z_{k}$ is made. The nature of the origin can be seen by looking at the submanifold $y=0$ in (A.3). The resulting metric is just the Fubini-Study metric on $C P^{n-1}$.

Acknowledgements. It is a pleasure to thank E. Calabi and S.-T. Yau for valuable advice, without which this paper could hardly have been written. Methods for constructing manifolds of SU(3) holonomy are discussed by S.-T. Yau in [18].

6 Mathematically, what is being considered here is the following. For any holomorphic vector bundle $T$, there is a natural mapping $H^{1}(T) \times H^{1}(T) \times H^{1}(T) \rightarrow H^{3}(T \times T \times T)$. If $T$ has structure group SU(3), there is a natural mapping $H^{3}(T \times T \times T) \rightarrow H^{3}(9) \simeq C$, where $\vartheta$ is the trivial line bundle and $C$ are the complex numbers. The composition of these operations gives a symmetric map $H^{1}(T) \times H^{1}(T) \times H^{1}(T) \rightarrow C$, which is indicated in Eq. (7.20) 


\section{References}

1. Green, M.B., Schwarz, J.H.: Anomaly cancellations in supersymmetric $D=10$ gauge theory and superstring theory. Phys. Lett. 149 B, 117 (1983)

2. Green, M.B., Schwarz, J.H.: Supersymmetrical dual string theory. Nucl. Phys. B 181, 502 (1981); Supersymmetric dual string theory (II.) Vertices and trees. Nucl. Phys. B 198, 252 (1982); Supersymmetrical dual string theory (III.) Loops and renormalization. Nucl. Phys. B 198, 441 (1982); Supersymmetrical string theories. Phys. Lett. 109 B, 444 (1982)

Green, M.B., Schwarz, J.H. Brink, L.: $N=4$ Yang-Mills and $N=8$ supergravity as limits of string theories. Nucl. Phys. B 198, 474 (1982)

3. Gross, D.J., Harvey, J., Martinec, E., Rohm, R.: Heterotic string. Phys. Rev. Lett. 524502 (1985). See also Freund, P.G.O.: Superstrings from 26 dimensions. Phys. Lett. 151 B, 387 (1985); Goddard, P., Olive, D.: In proceeding of the Conference on Vertex Operations in Mathematics and Physics, ed. J. Lepowsky et al. Berlin, Heidelberg, New York: Springer 1984

4. Candelas, P., Horowitz, G., Strominger, A., Witten, E.: Vacuum configurations for superstrings. Nucl. Phys. B (1985) (to appear)

5. Witten, E.: Symmetry breaking in superstring models and dimensional reduction of superstring models. Princeton preprints (1985)

6. Dine, M., Kaplunovsky, V., Mangano, M., Nappi, C., Seiberg, N.: Superstring model building. IAS preprint (1985)

7. Dine, M., Rohm, R., Seiberg, N., Witten, E.: Gluino condensation in superstring models. IAS preprint (1985)

8. Nepomechie, R., Wu, Yong-Shi, Zee, A.: University of Washington preprint (1985)

9. Cecotti, S., Derendinger, J.-P., Ferraro, S., Girardello, L., Roncadelli, M.: CERN preprint (1985)

10. Breit, J., Ovrut, B., Segre, G.: $E_{6}$ symmetry breaking in the superstring theory. University of Pennsylvania preprint (1985)

11. Sen, A.: Naturally light Higgs doublet in supersymmetric $E_{6}$ grand unified theory. Fermilab preprint (1985)

12. Georgi, H., Glashow, S.: Making do without the $t$ quark. Nucl. Phys. 167 B, 173 (1980)

13. Kane, G., Peskin, M.: A constraint from $B$ decay on models with no $t$ quark. Nucl. Phys. B 195, 29 (1982)

14. Griffiths, P., Harris, J.: Principles of Algebraic Geometry. New York: Wiley 1978

15. Calabi, E.: Ann. Sci. Ec. Norm. Super. 12, 266 (1979)

16. Eguchi, T., Hanson, A.S.: Asymptotically flat self-dual solutions to euclidean gravity. Phys. Lett. 74 B, 249 (1978)

17. Freedman, D.Z., Gibbons, G.W.: In Superspace and supergravity, p. 449. Cambridge: Cambridge University Press 1981

18. Yau, S.-T.: In: Proceedings of the Argonne Symposium on Anomalies. Geometry and Topology (to appear)

Communicated by A. Jaffe

Received April 29, 1985 
